\title{
Preliminary work of real-time ultrasound imaging system for 2-D array transducer
}

\author{
$\mathrm{Xu} \mathrm{Li}^{\mathrm{a}}$, Jiali Yang a and Mingyue Ding ${ }^{\mathrm{a}, \mathrm{b}}$ and Ming Yuchi ${ }^{\mathrm{a}}{ }^{\mathrm{*}}$ \\ ${ }^{a}$ Department of Biomedical Engineering, School of Life Science and Technology, Image Processing \\ and Intelligence Control Key Laboratory of Education Ministry of China, Huazhong University of \\ Science and Technology, 1037 Luoyu Road, Wuhan, 430074, China \\ ${ }^{b}$ Wuhan National Laboratory for Optoelectronics, Huazhong University of Science and Technology, \\ 1037 Luoyu Road, Wuhan, 430074, China
}

\begin{abstract}
Ultrasound (US) has emerged as a non-invasive imaging modality that can provide anatomical structure information in real time. To enable the experimental analysis of new 2-D array ultrasound beamforming methods, a pre-beamformed parallel raw data acquisition system was developed for 3-D data capture of 2D array transducer. The transducer interconnection adopted the row-column addressing (RCA) scheme, where the columns and rows were active in sequential for transmit and receive events, respectively. The DAQ system captured the raw data in parallel and the digitized data were fed through the field programmable gate array (FPGA) to implement the pre-beamforming. Finally, 3-D images were reconstructed through the devised platform in real-time.
\end{abstract}

Keywords: 2-D array transducer, 3-D imaging system, row-column scheme, real-time

\section{Introduction}

In the last decade, research investigators and commercial companies have further advanced ultrasound imaging with the development of 3D ultrasound [1-3]. The major reason for the increase in the use of 3D ultrasound is related to the limitations of $2 \mathrm{D}$ viewing of $3 \mathrm{D}$ anatomy, using conventional ultrasound. Several researchers have explored 3-D ultrasound imaging by mechanically translating a 1-D array to acquire multiple B-scans for offline processing of image reconstruction [4]. The mechanical translation may be done either by using motor or freehand methods. In both cases, lack of reliability and the slow capture speed limit the use of such methods for the slowness and. On the contrary, 2-D arrays have several advantages compared with mechanically scanned 1-D arrays. 2-D arrays do not require moving parts to acquire a 3-D volume to confirm that the beam is well focused in elevation at this depth. Furthermore, 3-D ultrasound (US) with a 2-D array can scan a cylindrical volume thus providing simultaneous longitudinal and transverse views of the test objects

\footnotetext{
* Address for correspondence: Ming Yuchi, Department of Biomedical Engineering, School of Life Science and Technology, Image Processing and Intelligence Control Key Laboratory of Education Ministry of China, Huazhong University of Science and Technology, 1037 Luoyu Road, Wuhan, 430074, China. Tel.: 13995547537; Fax: 027-87792366; E-mail: m.yuchi@gmail.com .
} 
without probe switching or manipulation, which reduces the scan time. As some research groups have shown their results of the 2-D array based 3-D system, the image quality is still poor [5]. To make an improvement of the image quality, we have built a system, which can be used for the beamforming methods research, to gain the pre-beamformed data of 2-D array based ultrasound imaging system at the pre-beamformed level in our previous work [6]. After that, we have revised the system and the transducer to improve the echo signal quality and we have also done some test object experiments for imaging in real-time.

\section{Methodology}

\subsection{Row-column addressing method}

We have designed the $32 \times 32$ 2-D array based on row-column addressing scheme (see Figure 1) [5].

The bottom layer consists of vertical electrodes (Figure 1(a)) and the top layer consists of horizontal electrodes (Figure 1(b)). In transmission, the bottom layer serves as the ground and the top layer was fired as the transmitters. In one top layer line, all elements fire simultaneously to emit a planar wave front and the reflected echoes are received in the vertical direction. With this method, the total number of physical connections is decreased from $\mathrm{N} \times \mathrm{N}$ to $2 \mathrm{~N}$.

\subsection{3-D ultrasound imaging system construction}

The 3-D ultrasound imaging system consists of three parts: an observation tank with a 2-D transducer, a set of data acquisition system and a computer controller for data storage, signal processing and visualizing. In the observation tank, the test object is placed at the depth of $5 \mathrm{~cm}$ in the center. A 2-D transducer at the center frequency of $8 \mathrm{MHz}$ was placed at precise position to capture the echo signals. The received signals are amplified, digitized and stored for off-line processing or signal processed for real-time image reconstruction and visualization.

The data acquisition electronics is a customized system supported by National Instrument (NI) for high-speed data capture. The system can capture the received signals by 4 NI 5752 boards, every board supports 32 channel data parallel capture with $50 \mathrm{MB} / \mathrm{s}$ sample rate and 14-bit A/D each channel. In NI 5752 modulus, the analog signals are processed with low-noise amplifier, variable-gain amplifier and anti-aliasing filter before digitization. The digital signals are transferred into the FPGA (NI PXIe-7966R) for de-serializing. All the 32-channel high-speed digitized signals are transferred to the backboard routing of computer controller NI PXIe-1085 RT. The NI LabView interface is used

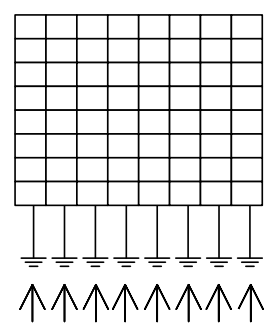

(a)

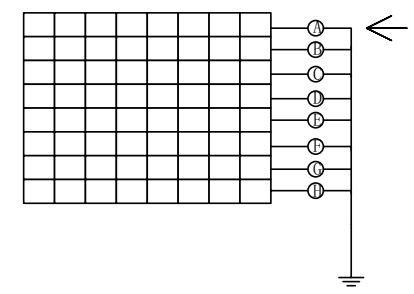

(b)

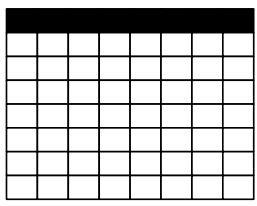

(c)

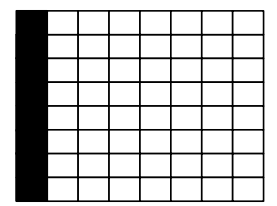

(d)

Fig. 1. Row-column addressing method. (a) The bottom layer. (b) The top layer (c) Transmit on top layer in the horizontal direction. (d) Receive on bottom layer in the vertical direction. 
to realize the system control, synchronization and imaging programming. The signals are processed for envelope detection, logarithm transformation interpolation, and receive focusing in the 7966R. Moreover, we have adopted a visualization toolkit to visualize our 3-D real-time results at the frame rate of 20 frames/s.

\section{Simulation and experiment results}

\subsection{Simulation}

Using the simplified scheme, point spread function was simulated with Field II (see Figure 2) [7]. The transmit aperture was set as an azimuthal pitch of $\lambda / 2=0.15 \mathrm{~mm}$ and elevation height of $32 \lambda / 2=4.8 \mathrm{~mm}$ in the row direction, while the receive aperture had an elevation pitch of $\lambda / 2=0.15 \mathrm{~mm}$ and an azimuthal length of $4.8 \mathrm{~mm}$ in the column direction and focus depth is $40 \mathrm{~mm}$. Gaussian pulses at the center frequency of $8 \mathrm{MHz}$ and a $-6 \mathrm{~dB}$ fractional bandwidth of $50 \%$ were used for excitation to verify the adopted method and from the simulation results the $-6 \mathrm{~dB}$ main lobe width is $2.26 \mathrm{~mm}$.

\subsection{Transducer}

A $32 \times 322$-D array transducer at the inch of $1 \mathrm{~cm} \times 1 \mathrm{~cm}$ was made (Figure 3 ). The $-6 \mathrm{~dB}$ fractional bandwidth was measured as $60 \%$ at the center frequency of $8 \mathrm{MHz}$. The transducer enclosure was fabricated by $3-\mathrm{D}$ printer.

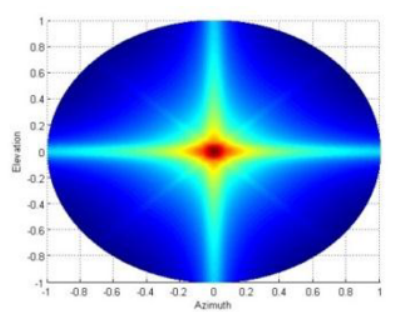

(a)

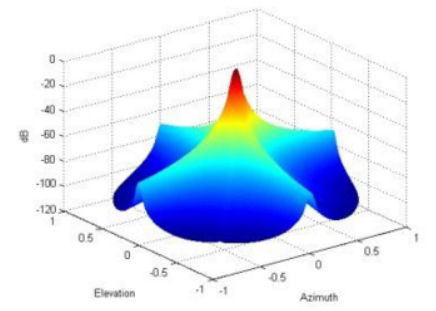

(b)

Fig. 2. Simulated beamplots of the row-column transducer: (a) 3-D beamplot; (b) contour plot with lines at $-10,-20,-30,-40$, and $-50 \mathrm{~dB}$.
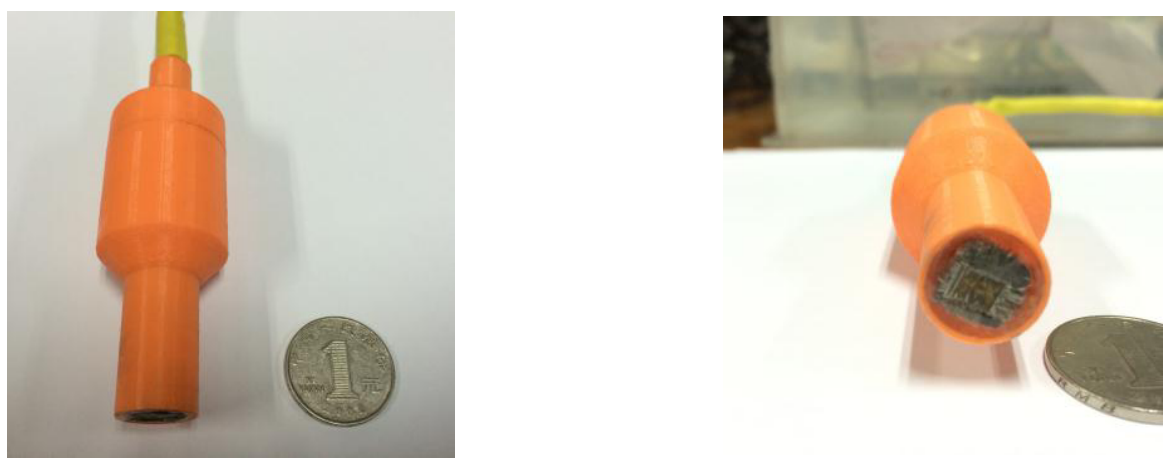

Fig. 3. The 2-D array transducer. 


\subsection{Experiment}

The system prototype is shown in Figure 4. Figure 4(a) shows the ultrasound transmit and multiplexer for the pulse signal generation. Figure 4(b) is the power supply with noise lower than 30 mv p-p. Figure 4(c) is the NI high-speed data capture system. Figure 4(d) shows the transducer and the test object, we have set a strong-reflection steel plate at the depth of $5 \mathrm{~cm}$ for the echo signal is weak now.

Figure 5 shows the captured ultrasound echo signals in the LabView interface. After the process of receive focusing, envelope detection, logarithm transformation, DAS (delay and sum) and image interpolation we successfully reconstructed the 3-D image in real-time (Figure 6).

We have done some further work about the spatial resolution measurement of the built array system. To measure the vertical resolution, we have set the phantom with different vertical spacing of two

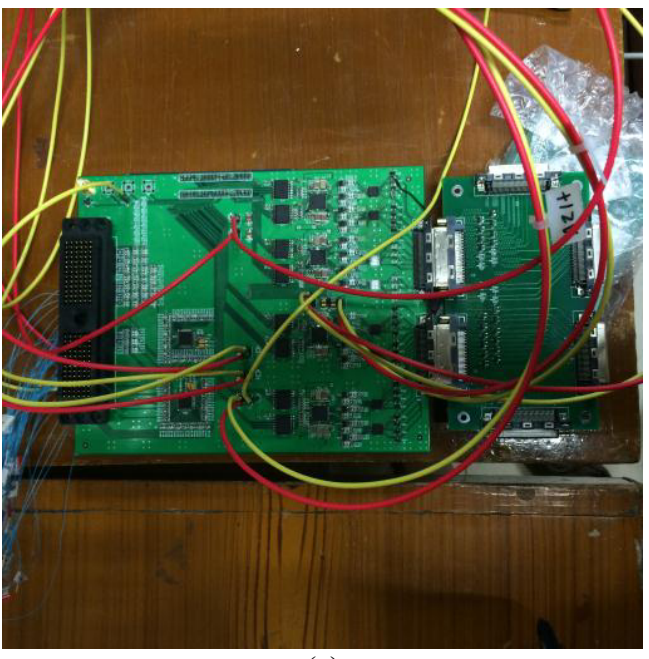

(a)

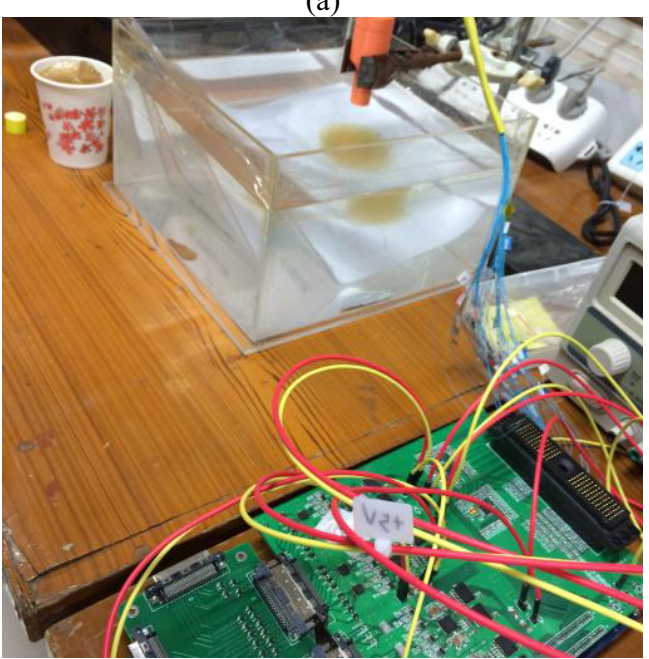

(c)

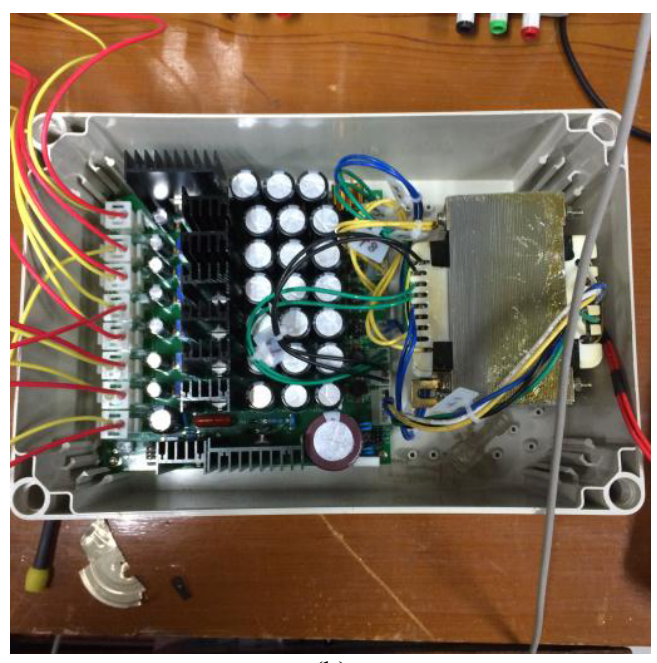

(b)

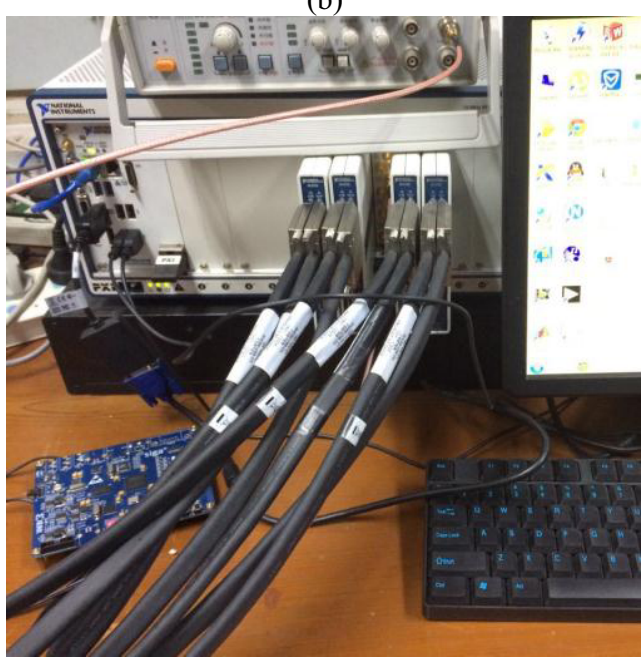

(d)

Fig. 4. Prototype of the real-time ultrasound imaging system (a) transmit and multiplexer (b) power supply (c) NI data capture system (d) transducer and test object. 


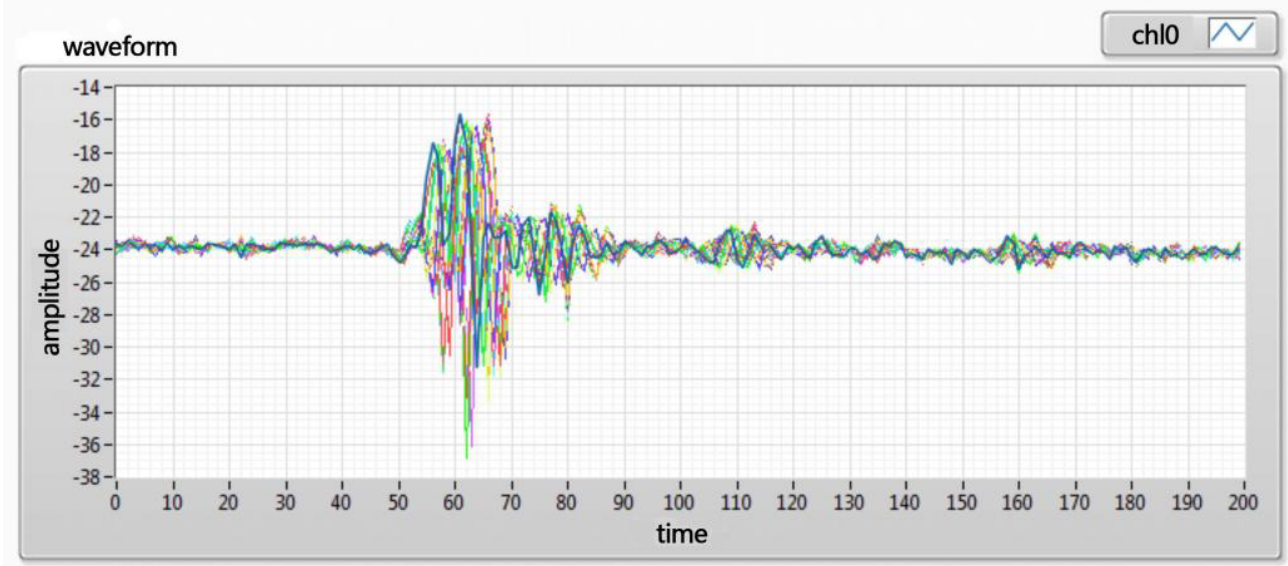

Fig. 5.32 channels of echo signals.

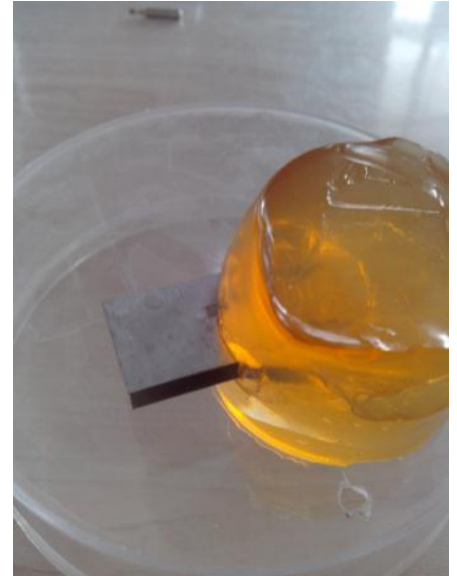

(a)

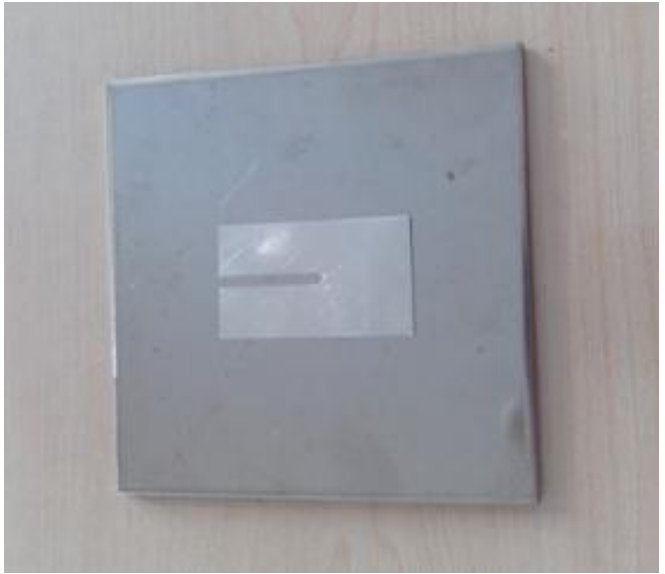

(b)

Fig.6 (a) The phantom of 2 strong-reflections at the spacing of $1.2 \mathrm{~mm}$. (b) The phantom of 2 strong-reflections at the spacing of $0.27 \mathrm{~mm}$.

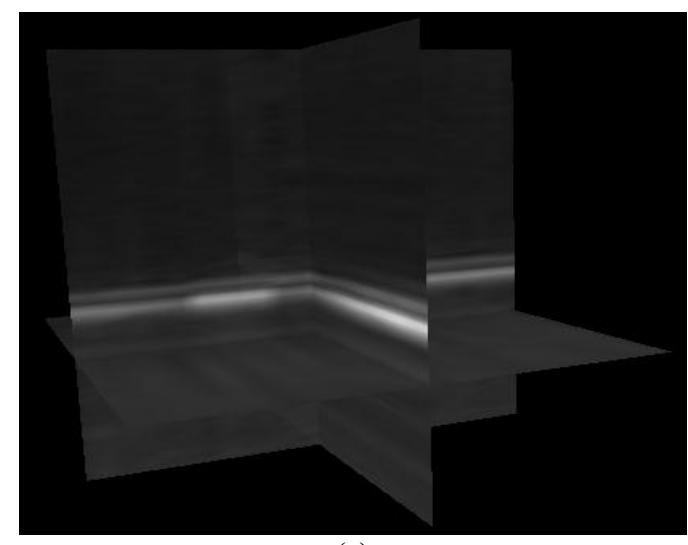

(a)

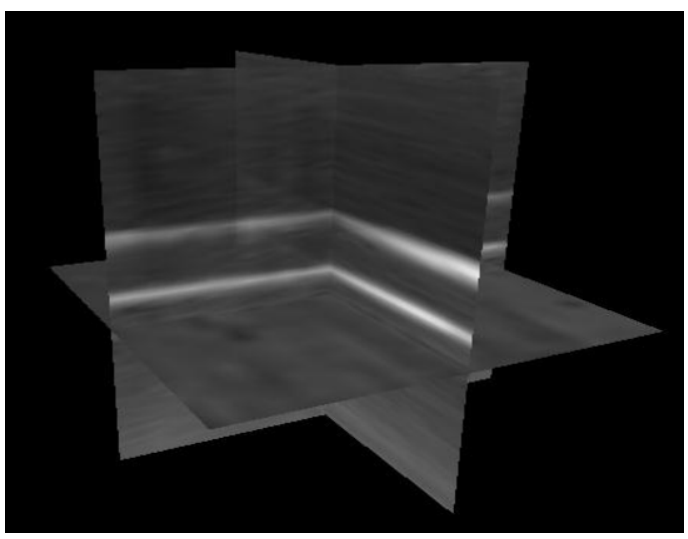

(b)

Fig. 7. The reconstructed 3D results of phantom in Figures 6(a) and 6(b). 


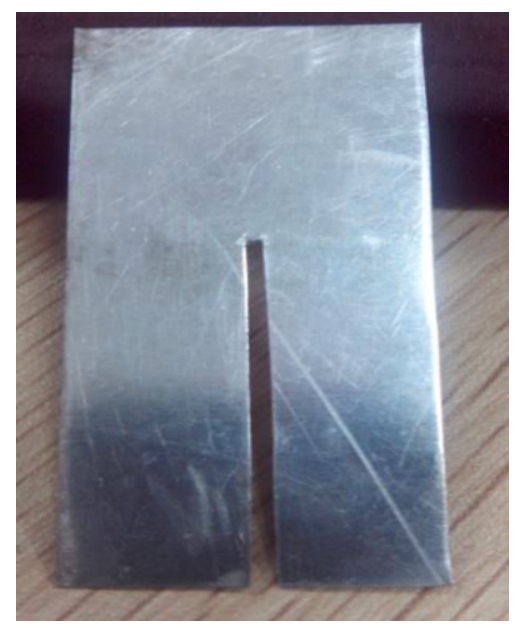

Fig. 8. The phantom of a strong reflection surface with a stripped opening of $1.5 \mathrm{~mm}$ width.

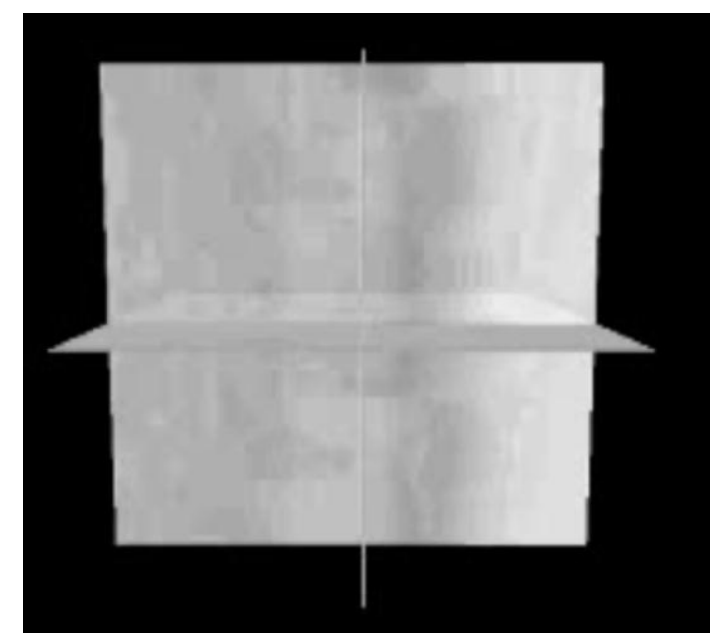

Fig. 9. The reconstructed 3D results of phantom in Figure 8.

strong reflection surfaces in Figures 6(a) and 6(b). Figures 7(a) and 7(b) show the corresponding reconstructed images. It can be found that the two surfaces can be distinguished, which means the vertical resolution can reach $0.27 \mathrm{~mm}$. To measure the lateral resolution, we have built the phantom of a strong reflection surface with a stripped opening of $1.5 \mathrm{~mm}$ width (Figure 8), which can be distinguished in the reconstructed 3D result in Figure 9.

\section{Discussion}

2-D array based 3-D ultrasound system can overcome the disadvantage of 2-D one of the subjectivity of the conventional exam [8], which results from the dependence on the experience and knowledge of the diagnostician to manipulated the ultrasound transducer, mentally transform the 2-D images into a 3-D tissue structure. The preliminary work has shown that we have successfully fabricated the 2-D ultrasound transducer and finished the construction of the whole system. But as the scan region of the transducer is small, echo signal is weak to capture for soft tissues now, we have only done the imaging results of strong-reflection test objects such as steel plate in the gel. And different test object experiments are implemented in the future to form real-time soft tissue image results.

\section{Conclusions}

A preliminary real-time ultrasound imaging system was presented in this paper. We designed the 2-D array transducer with the simplified method of row-column scheme, transmit and multiplexer circuit, the capture and visualization interface with the NI capture system. The simulation and experiment results were also shown.

Future work includes experiment on different testing objects and improving the image quality. 


\section{Reference}

[1] L. Masotti, E. Biagi, M. Scabia, A. Acquafresca, R. Facchini, A. Ricci and D. Bini, FEMMINA real-time, radio-frequency echo-signal equipment for testing novel investigation methods, IEEE Transactions on Ultrasonics, Ferroelectrics, and Frequency Control 53 (2006), 1783-1795.

[2] S. Ricci, E. Boni, F. Guidi, T. Morganti and P. Tortoli, A programmable real-time system for development and test of new ultrasound investigation methods, IEEE Transactions on Ultrasonics, Ferroelectrics, and Frequency Control 53 (2006), 1813-1819.

[3] H.J. Hewener, H.J. Welsch, C. Gunther, H. Fonfara, S.H. Tretbar and R.M. Lemor, A highly customizable ultrasound research platform for clinical use with a software architecture for 2D-/3D-reconstruction and processing including closed-loop control, World Congress on Medical Physics and Biomedical Engineering, Munich, Germany, 2009, pp. 342-345.

[4] A. Fenster and D.B. Downey, 3-D ultrasound imaging: A review, IEEE Engineering in Medicine and Biology 15 (1996), $44-49$.

[5] H.S. Chi and J.T. Yen, A $256 \times 2562$-D array transducer with row-column addressing for 3-D rectilinear imaging, IEEE Transactions on Ultrasonics, Ferroelectrics, and Frequency Control 56 (2009), 837-847.

[6] X. Li and Y.C. Ming, A preliminary work on pre-beamformed data acquisition system for ultrasound imaging with 2-D transducer, SPIE Medical Imaging, International Society for Optics and Photonics, San Diego, 2014, 90400J.

[7] J.A. Jensen and N.B. Svendsen, Calculation of pressure fields from arbitrarily shaped, apodized, and excited ultrasound transducers, IEEE Transactions on Ultrasonics, Ferroelectrics, and Frequency Control 39 (1992), 262-267.

[8] A. Fenster and D.B. Downey, 3-D ultrasound imaging: A review, IEEE Engineering in Medicine and Biology 15 (1996), 44-49. 